\title{
Implementation of Modified Wiener Filtering in Frequency Domain in Speech Enhancement
}

\author{
Mr.C.Ramesh Kumar ${ }^{1}$, Dr. M.P.Chitra ${ }^{2}$ \\ Research Scholar' Sathyabama Institute of Science and Technology ${ }^{1}$ \\ Department of Electronics and Communication Engineering ${ }^{1}$ \\ Assistant Professor, Panimalar Engineering College, Chennai ${ }^{1}$ \\ Professor, Department of Electronics and Communication Engineering ${ }^{2}$ \\ Panimalar Institute of Technology, Chennai, Tamil Nadu, India ${ }^{2}$
}

\begin{abstract}
The most common complaint about Digital Hearing Aids is feedback noise. Many attempts have been undertaken in recent years to successfully reduce feedback noise. A wiener filter, which calculates the wiener gain using before and after filtering SNR, is one technique to reduce background noise. Modified Noise Reduction Method (MNRM), a new way for reducing feedback noise Reduction, is presented in this work. In the Modified Noise Reduction Strategy, the advantages of a wiener filter are merged with a decision-directed approach and a twin-stage noise suppression technique The Modified Noise Reduction method can reduce the noise more successfully, according to comprehensive MATLAB programming, investigation, and findings analysis. After being modelled in MATLAB for seven distinct noise types, the SNR of the two architectures is compared.
\end{abstract}

Keywords-Digital hearing aid; least mean square value; noise reduction method; power spectral density

\section{INTRODUCTION}

The main purposes of DHA is to give frequency dependent amplification to persons who are deaf or hard of hearing. The DHA may also filter background noise, compress dynamic range, and remove feedback because it has an unit for external voice processing. Because digital hearing aids require frequency dependent amplification, sub band domain signal processing is a preferable option. When compared to the block transform, the lapped transform produces a bigger rejection of side lobes [22]. DFT, DCT, and MLT, AMLT, among others, are examples of block transformation techniques, whereas MLT, AMLT, and other lapped transformation techniques are examples of lapped transformation techniques [17].

DHA feedback can be cancelled using the LMS or NLMS algorithms. Because the NLMS algorithm has variable step size control, it has a better steady state behavior than the LMS algorithm. The two solutions proposed outperform Speech improvement using a decision-driven approach while retaining the decision-driven approach's advantages. The advantages of contemporary sub band domain digital hearing aid systems were explored by Ashutosh et al (2011) [1,2]. Easy gain adjustments in each sub band, very quick convergence of adaptive filters, and calculation savings are just a few of the benefits.
In today's sub band domain Digital Hearing Aids, the wiener filter is employed to reduce noise [3]. Feedback elimination is done using the NLMS approach. NLMS algorithm enhances active noise reduction, resulting in more consistent gain. To suppress feedback, adaptive filters in modern sub band DHA use block transformations like as DFT, GDFT. The adaptive filter techniques LMS and NLMS are used to eliminate the feedback noise by varying the filter coefficients [6]. Error e(n) provides the smallest term, the filter co-efficient is thought to be the best The co-efficient of these filters are utilised to predict constantly in the forward path and cancel the FB path $[4,5]$. Ear may be harmed if the increased speech volume surpasses the upper thresholds of hearing, hence DRC should be included in the hearing aid to avoid painful listening. Background noise suppression is the basic function of speech enhancement in DHA. Hearing aid users will benefit from improved unwanted sound suppression techniques that will improve performance and provide a more pleasant listening experience.

Multichannel DRC with decoded Digital Frequency Warping to eliminate noise, the DFW filter was replaced by an all-pass filter.[9] To compensate for hearing loss in digital hearing aids, the filter bank channel gains must be changeable over a wide dynamic range[10,11].In Biological basic DHA contain two channel, the first channel includes a directional unit for receiving the acoustic input signal and providing a directional signal; a correlative unit for receiving the directional signal and providing a noise reduced signal $[12,13]$.Compare to 16 point TAP wiener filter,32 point TAP wiener filter cancels $17 \mathrm{db}$ noise [14]. A delay-based NLMS technique is used to update the two adaptive filters $[15,16]$. Internal and external Acoustic feedback noise reduction using DSP Processor. Various technological and acoustic modification approaches are available to suppress or lesser auditory feedback. $[18,19]$. The effect of setting a hearing aid's gain control to a level just below that required to cause audible oscillation was explored [23,24]. Multi-channel compression techniques provide a realistic way to transfer speech signals' large dynamic range onto the reduced dynamic range of hearing-impaired listeners [25]. In DHA to regulate the gain value with different frequency and improve the SN [26]. Preprocessing filter algorithm used to High SNR with low noise speech signal produced in industry environment [27]. 


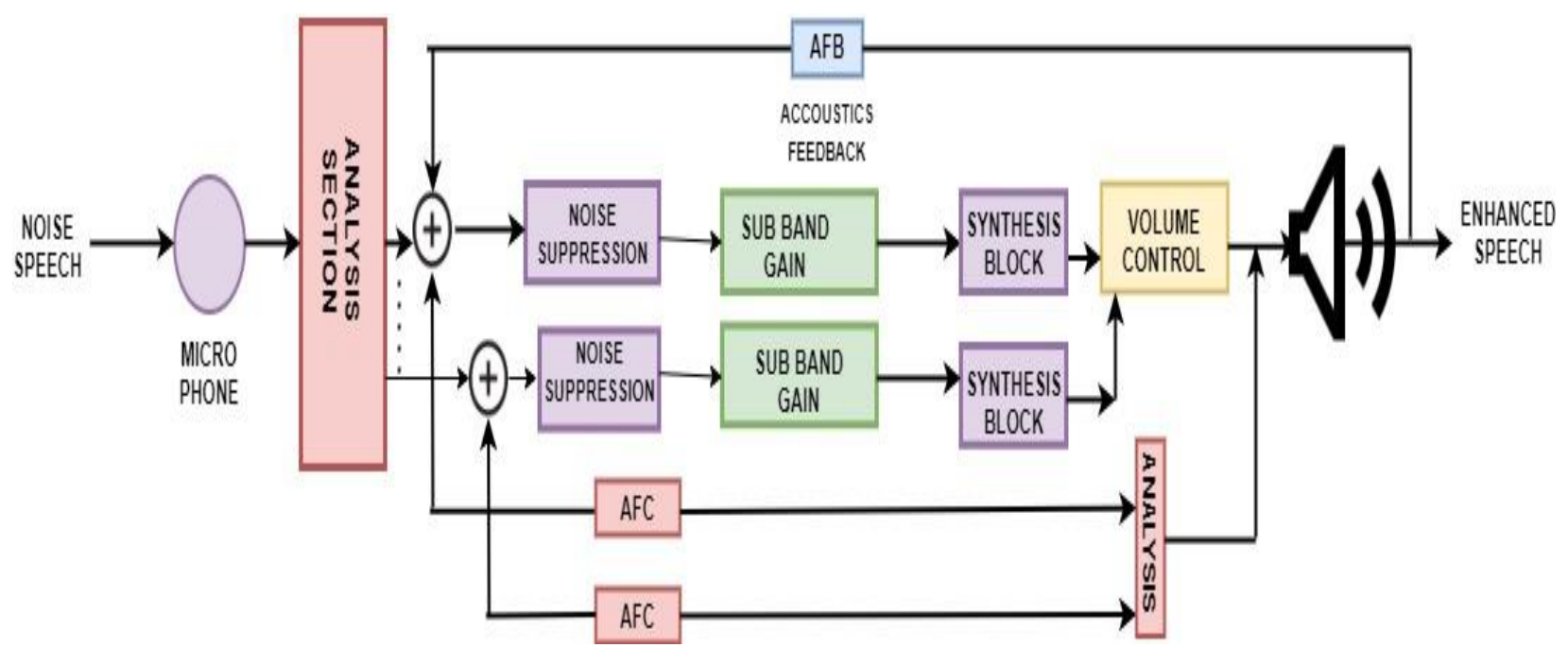

Fig. 1. Functional Diagram of Improved Sub Band Domain DHA.

A Wiener filter is used to reduce noise in most improved sub band domain digital hearing aids [7,8]. A block schematic of a modern sub band domain DHA is shown in Fig. 1. It works with the block transform's created subbands. As input to the DHA, the feedback passage signal from the AFB channel is combined with the noisy speech. Signal processing is used for hearing-impaired correction, noise elimination, AFB elimination, and DRC $[20,21]$. The analysis portion generates the appropriate subbands, which are then reproduced in the synthesis section.

This paper's structure is as follows: The first section contains a brief introduction. The Modified Subband Domain Digital Hearing Aid is shown in Section II. Sections A and B detail the Wiener Filter and MNRM algorithms. Section III concludes with the results, debates, and conclusions.

\section{TRADITIONAL WIENER APPROACH}

The Wiener filter is a well-known signal augmentation method that has been applied to a variety of applications. The Wiener filter's basic concept is to separate noise eliminated original speech from the noise mixed speech. Approximation is made by minimising the Mean. MSE between the desired signal $S(n)$ and the error signal e(n). As a result of this optimization's frequency domain solution, the following filter transfer function is obtained.

$H(W)=\frac{P_{S}(W)}{P_{S}(W)+P_{V}(W)}$

$\mathrm{Ps}(\mathrm{W})$ - PSD of noiseless speech and $\mathrm{Pv}(\mathrm{W})$-PSD of noise added Speech.

The SNR is defined as follows by [13]:

$H(W)=\frac{P_{S}(W)}{P_{V}(W)}$

$\mathrm{H}(W)$ can be Expressed in wiener Filter as follows:

$\mathrm{H}(W)=\left[1+\frac{1}{\text { Signal to Noise Ratio }}\right]^{-1}$
The Wiener filter has the disadvantage of having a constant frequency measures at every frequency and requiring calculation of the clean signal and noise power spectral density before filtering. This section shows how to create an AWF that uses the changing local statistics of the speech signal. The proposed technique is depicted in Fig. 1 as a block diagram. In this procedure, the expected mean value of speech signal $\mu_{\mathrm{x}}$ and $\operatorname{Var}{\sigma_{x}}^{2}$ are employed With a variance of $\sigma_{x}$, the added noise $\mathrm{v}(\mathrm{n})$ is said to have $\mu_{x}=0$ and a white nature. As a result, we may take the Power Spectrum $P_{V}(w)$ as follows:

$P_{V}(w)=\sigma_{V}^{2}$

A brief part of a voice transmission in which the input $\mathrm{x}(\mathrm{n})$ is regarded stationary.

$\mathrm{x}(\mathrm{n})=\mu_{x}+\sigma_{x} w(n)$

The Mean $\mu_{x}$ and SD of $\sigma_{x}$ are represented by, A noise with a unit variance is called a unit variance noise $w$ (n)The Wiener filter transfers inside this tiny section of speech. The following functions can be used to approximate the function:

$H(w)=\frac{P_{S}(w)}{P_{S}(w)+P_{V}(w)}=\frac{\sigma_{S}^{2}}{\sigma_{S}^{2}+\sigma_{V}^{2}}$

Eq. (12) may be used to compute the Wiener filter's impulse response because $\mathrm{H}(\omega)$ is constant for this little portion of speech:

$H(w)=\frac{\sigma_{S}^{2}}{\sigma_{S}^{2}+\sigma_{V}^{2}} \delta(n)$

Eq. (13) can be used to represent the improved voice signal $\mathrm{s}(\mathrm{n})$ in this local field.

$$
\begin{aligned}
& \hat{S}(\mathrm{n})=\mu_{x}+\left(\mathrm{x}(\mathrm{n})-\mu_{x}\right) * \frac{\sigma_{S}^{2}}{\sigma_{S}^{2}+\sigma_{V}^{2}} \delta(n) \\
& =\mu_{x}+\frac{\sigma_{S}^{2}}{\sigma_{S}^{2}+\sigma_{V}^{2}}\left(\mathrm{x}(\mathrm{n})-\mu_{x}\right)
\end{aligned}
$$

We can say: if we update $\mu_{x}$

$\hat{S}(\mathrm{n})=\mu_{x}+\frac{\sigma_{S}^{2}}{\sigma_{S}^{2}+\sigma_{V}^{2}}\left(\mathrm{x}(\mathrm{n})-\mu_{x}(\mathrm{n})\right)$ 
Eq. (10) modifies the mean $\mu_{x}(\mathrm{n})$ and $\left(\mathrm{x}(\mathrm{n})-\mu_{x}(\mathrm{n})\right)$ independently from section to section before combining the results. The result signal $s(n)$ will be predominantly owing to $\mathrm{x}(\mathrm{n})$ if $\sigma_{S}^{2}$ is significantly larger than $\sigma_{V}^{2}$, and $\mathrm{x}(\mathrm{n})$ cannot be changed. When $\sigma_{S}^{2}$ is less than $\sigma_{V}^{2}$, the filtering effect is applied. When $\mu_{V}$ is zero, you'll notice that $\mu_{x}$ is the same as $\mu_{S}$. As a result, we may calculate $\mu_{x}(\mathrm{n})$ in Eq. (11) using $\mathrm{x}(\mathrm{n})$ by.

$\widehat{\mu_{S}}(\mathrm{n})=\widehat{\mu_{x}}(\mathrm{n})=\frac{1}{2 M+1} \sum_{K=n-M}^{n+M} x(k)$

$\sigma_{S}^{2}(\mathrm{n})=\left\{\begin{array}{c}\sigma_{x}^{2}(n)-\sigma_{V}^{2}(n) ; \text { if } \sigma_{x}^{2}(n)>\sigma_{V}^{2}(n) \\ 0 ; \text { Other wise }\end{array}\right.$

$(2 \mathrm{M}+1)$ samples were used in the estimation in the brief part. We need to calculate the speech by.

$\sigma_{S}^{2}=\sigma_{S}^{2}-\sigma_{V}^{2}$

calculate the signal variance $\sigma_{S}^{2}$ as follows from $\mathrm{x}(\mathrm{n})$ :

$\sigma_{x}^{2}(\mathrm{n})=\frac{1}{2 M+1} \sum_{K=n-M}^{n+M}\left(x(k)-\widehat{\mu_{x}}(n)\right)^{2}$

\section{A. Conventional Noise Suppression Technique using Wiener Filter}

This filter is used to reduce background noise. Wiener filter reduces noise by increasing the gain by the noisy voice input. The operations involved in wiener filtering noise reduction are depicted in Fig. 2. A noisy speech signal is fed into the Wiener filter, which separates it into $\mathrm{N}$ frames. FFT is used to convert time domain to frequency domain output. To achieve the high throughput and reduce the area instead of conventional FFT $\mathrm{R} 2{ }^{2} \mathrm{SDF}$ FFT is proposed in this work. PSD is calculated using noise characteristics. The initial silent area is used to calculate the noise characteristics of voice communications. The wiener gain is increased by the noisy voice signal in the frequency nature after it has been calculated.

The overall gain of the noisy voice signal is reduced to achieve noise reduction. Because the short temporal energy of a noisy signal is smaller than that of a speech signal, the noise signal is suppressed even after the noise suppressed speech signal is enhanced. Linear Filtering can be achieved by using Overlap Add Method.

\section{Overlap Add Method (Linear Filtering)}

Steps to compute overlap add:

- Use either SI (MKS) or CGS as primary units. (SI units are encouraged.) English units may be used as secondary units (in parentheses). An exception would be the use of English units as identifiers in trade, such as "3.5-inch disk drive".

- Divide the long sequence signal into L-length small sequence.

- Insert zeros into this small sequence, increasing the size of the new sequence to $m+n$, since $m+n=2^{n}$.

- Calculate the FFT of the full sequence, including the padding zeros.

- To resynthesize the signal, utilise IFFT and overlapping and adding the result.

\section{B. Proposed Modified Noise Reduction Method (MNRM)}

The modified noise reduction method operates in the frequency field and combines the qualities of a Wiener filter based on a decision-directed approach with a twin-stage noise suppression technique Fig. 3 depicts the activities involved in the Modified Noise Reduction Method (MNRM) for digital hearing aids. A noisy speech signal is fed into the INST filter, which separates it into $\mathrm{N}$ frames. FFT is used to convert time domain to frequency domain output. To achieve the high throughput and reduce the area instead of conventional FFT $\mathrm{R} 2^{2} \mathrm{SDF}$ FFT is proposed in this work. Noise characteristics are calculated from the first few frames. PSD is calculated using noise characteristics. The initial gain is determined in the same way as the Wiener filter gain after the pre and Post SNRs have been calculated. The cost of the MNRM is minimised by treating the posteriori and priory signal to noise ratio as the wiener filter gain, because the DHA must be operated in real time. $\mathrm{N}$ frames are created from the signal.

Because the MNRM's computing complexity is comparable to that of the wiener filter for noise removal, the proposed MNRM method can operate in real time, providing a better and more comfortable listening experience for hearing impaired people.
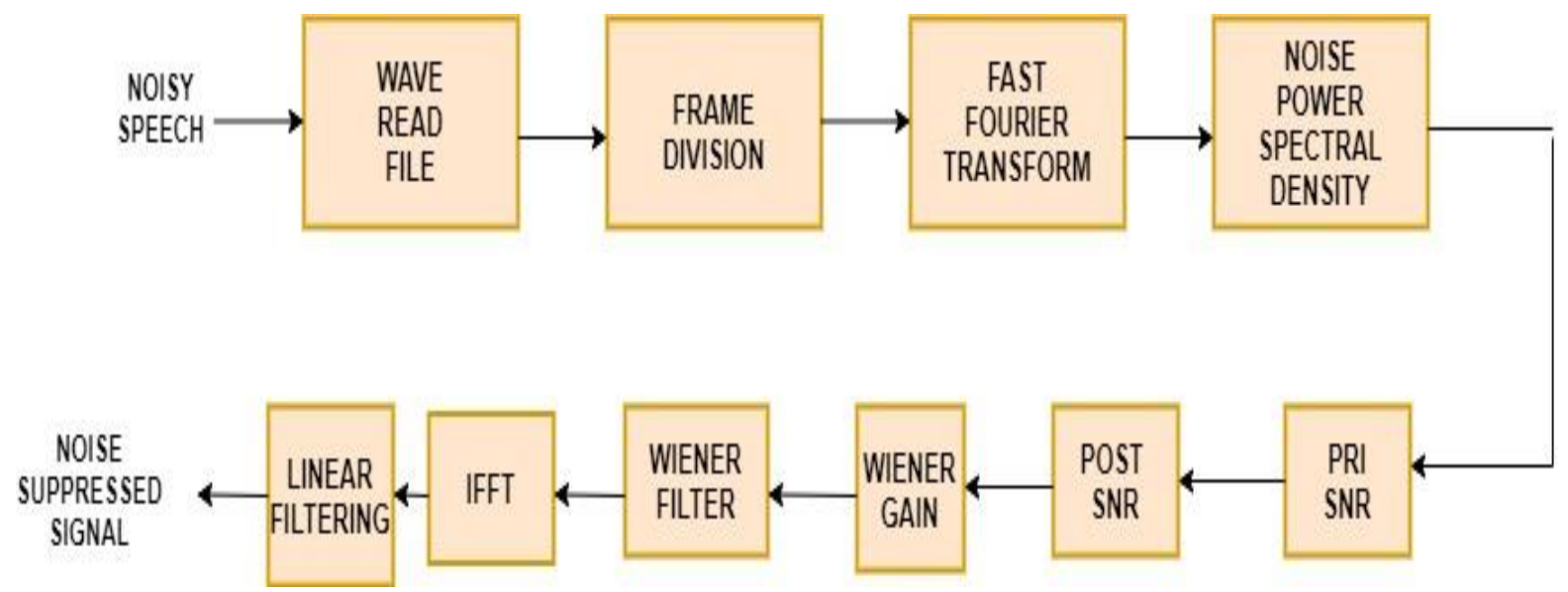

Fig. 2. Noise Reduction by Wiener Filter in DHA. 


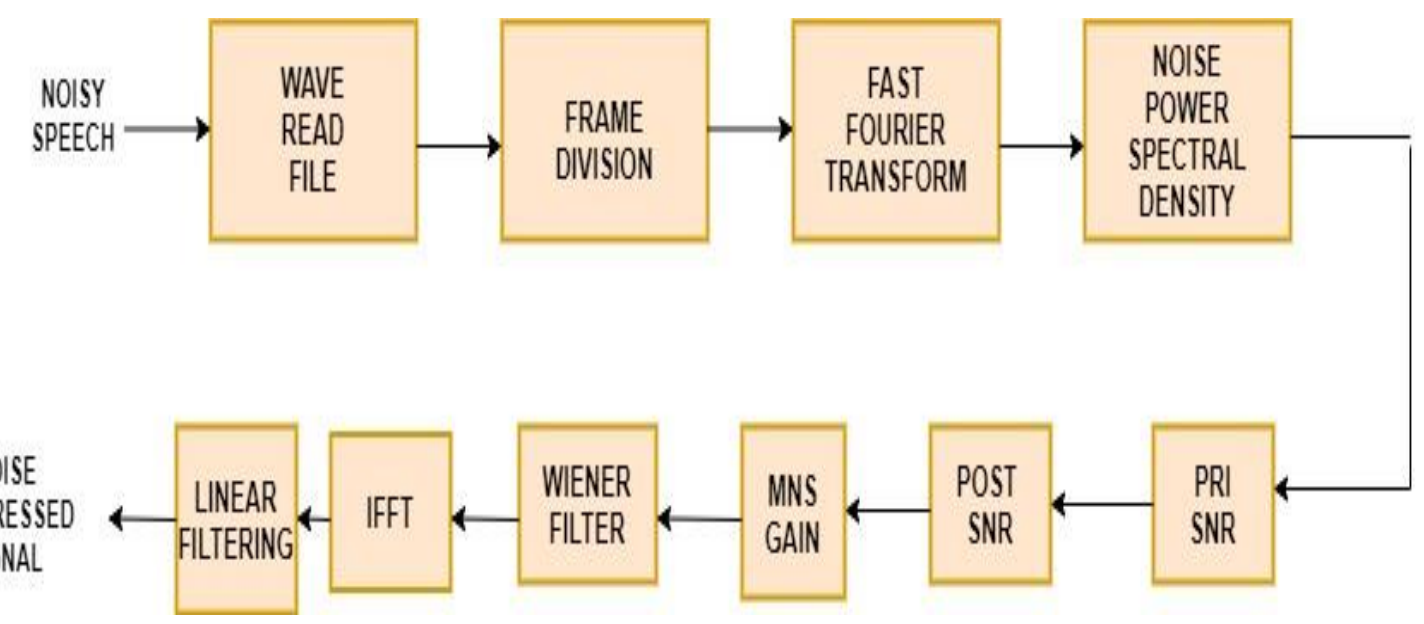

Fig. 3. Modified Noise Reduction Method in DHA.

\section{EXPERIMENTAL RESULTS AND DISCUSSION}

In MATLAB, we replicated a modern DHA with sub band domain with Compensation for hearing loss, DRC, noise reduction, and feedback cancellation for comparison. Four sub bands (S1) and eight sub bands (S2) are used in the two sets of $\mathrm{SP}$ in the sub band field. The research was undertaken performed on a variety of background noisy voice signals from the NOIZEUS database. The signals used to calculate the test signals the DHA performance are 8 sets of noisy speech sounds at varying SNRs such as $0 \mathrm{~dB}, 5 \mathrm{~dB}, 10 \mathrm{~dB}$, and $15 \mathrm{~dB}$. Fig. 6 depicts the performance of MNRM and wiener filtering approaches for noise suppression in DHA applications. We tried two ways using the identical type of loud voice signals for both methods for the sake of performance testing.
Fig. 4 shows the noise elimination of wiener filtering. Left side represents Time domain signal for clean speech, Noisy speech with street Noise and Enhanced Speech using Wiener and right side represent the Spectrogram for Clear, Noisy and Enhanced speech using Wiener. Fig. 5 shows the noise elimination of MNRM filter. Left side represents Time domain signal for clean speech, Noisy speech with street Noise and Enhanced Speech using MNRM and right side represent the Spectrogram for Clear, Noisy and Enhanced speech using MNRM filter. The evaluation of the result is computed by adding the absolute amplitude of the improved voice signal at the outcomes of the DHA. The figure's analysis in some trails, Fig. 6 reveals that the MNRM has a slight advantage over the wiener filter in terms of noise suppression.

The SNR obtained with various Ambient noises is compared for both approaches are shown in Table I.
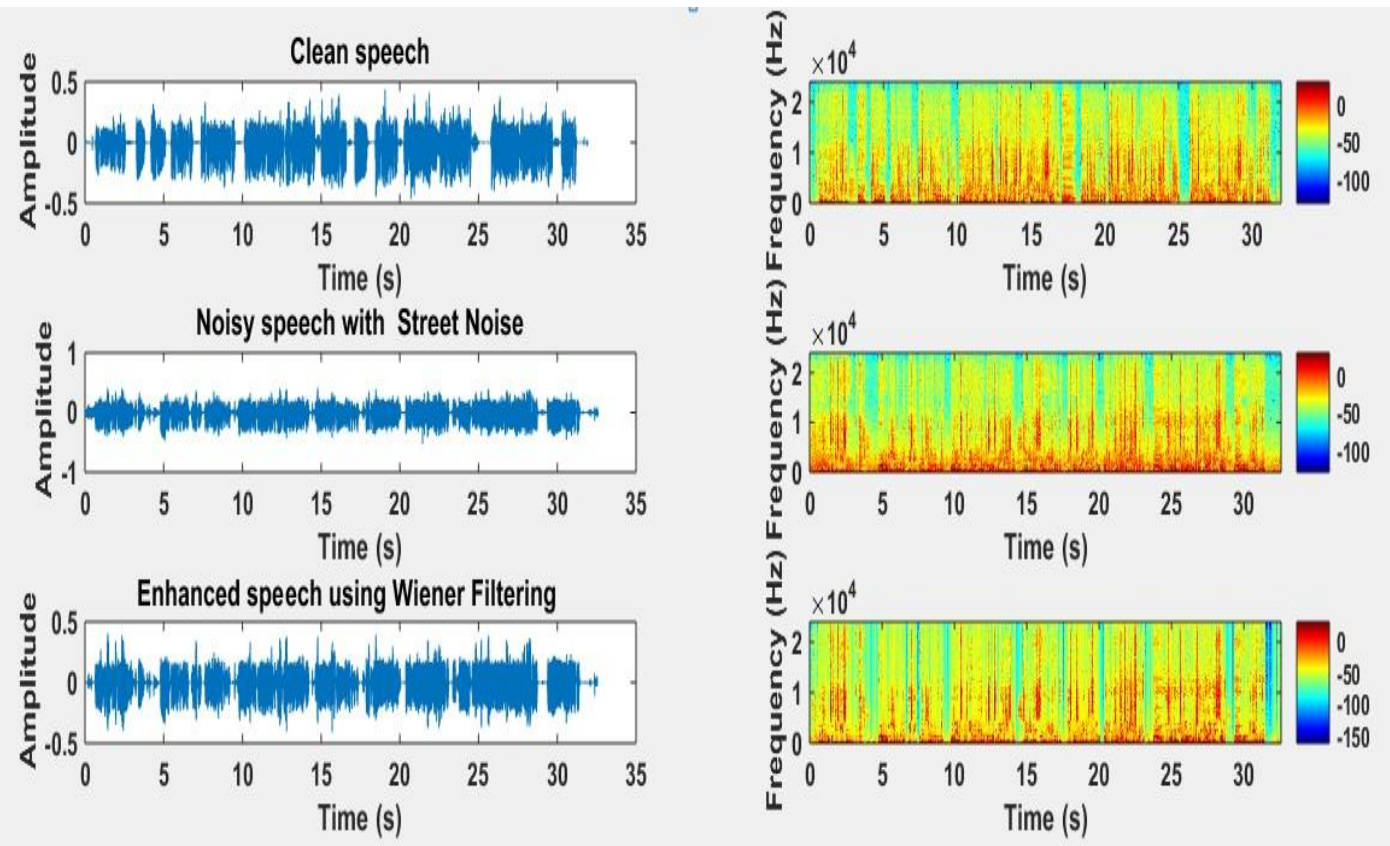

[]

Fig. 4. Left Side represents Time Domain Signal for Clean Speech, Noisy Speech with Street Noise and Enhanced Speech using Wiener and Right Side represent the Spectrogram for Clear, Noisy and Enhanced Speech using Wiener. 

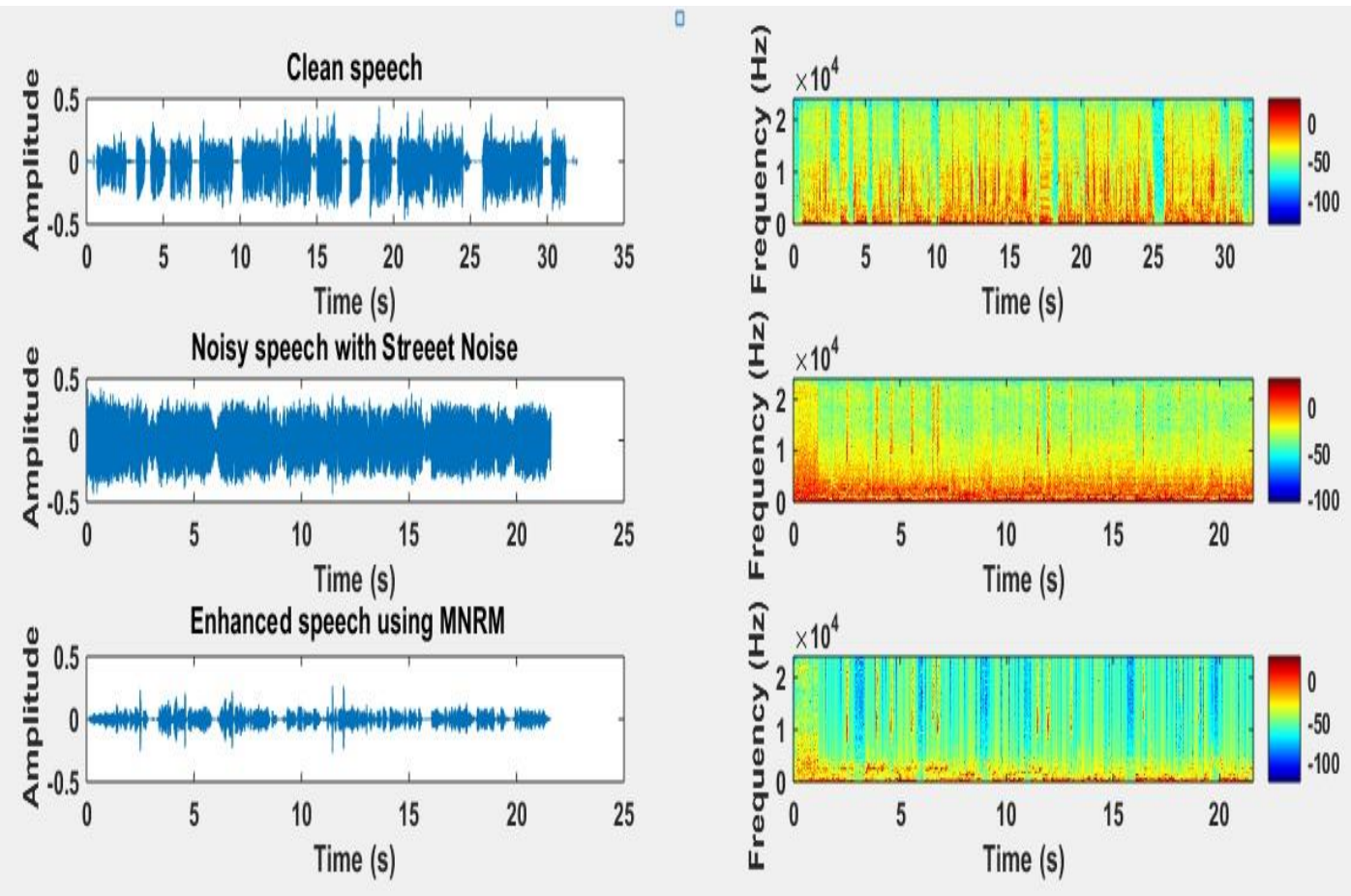

Fig. 5. Left Side represents Time Domain Signal for Clean Speech, Noisy Speech with Street Noise and Enhanced Speech using MNRM and Right Side represent the Spectrogram for Clear, Noisy and Enhanced Speech using MNRM.

\section{SNR COMPARISON OF EXISTING WIENER AND PROPOSED MNRM}

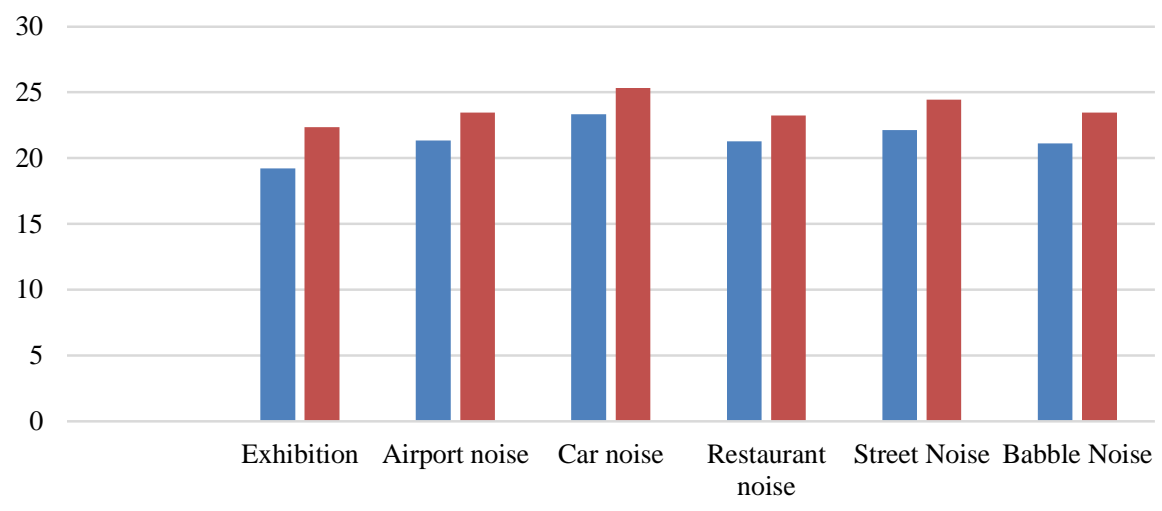

匹NR by existing Wiener Filtering $(\mathrm{db}) \quad$ - SNR by proposed MNRM $(\mathrm{db})$

Fig. 6. SNR Comparison of Wiener and MNST Filters.

TABLE I. COMPARING SNR OF WIENER FILTERING AND MNRM

\begin{tabular}{|l|l|l|}
\hline Various Ambient noises & SNR by existing Wiener Filtering $(\mathbf{d b})$ & SNR by proposed MNRM $(\mathbf{d b})$ \\
\hline Exhibition & 19.234 & 22.345 \\
\hline Airport noise & 21.345 & 23.456 \\
\hline Car noise & 23.345 & 25.345 \\
\hline Restaurant noise & 21.267 & 23.234 \\
\hline Street Noise & 22.145 & 24.456 \\
\hline Babble Noise & 21.134 & 23.456 \\
\hline
\end{tabular}




\section{CONCLUSION}

This research developed a novel approach for suppressing background noise in DHA via signal processing. In Matlab simulations, the performance of MNRM is compared to that of the wiener filter. In this work concluded with seven different Ambient noises (Exhibition, Airport, Car, Restaurant, Street, Babble) compare with Wiener and MNRM filters. Based on the comparison these two filters for the SNR ratio for those different noises increased with our proposed model of MNST filter. Approximately 14 to $15 \%$ of SNR ratio increased by using with proposed model. The MNST can be employed in modern subband domain DHA for noise reduction, as shown in Matlab. The user of hearing aids will benefit from this, with a better and more comfortable listening experience.

\section{REFERENCES}

[1] Ashutosh Pandey, V. John Mathews,"Low-Delay Signal Processing for Digital Hearing Aids", IEEE Trans. Audio,Speech and Language Processing, 19, pp. 699-710, 2011.

[2] Hellgren J, "Analysis of feedback cancellation in hearing aids with filtered-X LMS and the direct method of closed loop identification", IEEE Trans. Speech Audio Process., 10, pp.119-131, 2002.

[3] Philips, C. Loizou, "Speech Enhancement Theory and Practice", CRC Press.

[4] Siqueira M. G., Alwan A.,"Steady-state analysis of continuous adaptation in acoustic feedback reduction systems for hearing-aids", IEEE, Trans. Speech Audio Process., 8, pp.443-453, 2000.

[5] Stone M. A., Moore B. C. J.,"Tolerable hearing aids delays.II: Estimation of limits imposed during speech production",J. Amer. Academics of Audiology, 11, pp. 325-338, 2002.

[6] Sunitha S. L., Udayashankara V.,"Fast Factored DCTLMS Speech Enhancement for Performance Enhancement of Digital Hearing Aid", World Academy of Science, Engineering and Technology, 10, pp. 253257, 2005.

[7] Wyrsch S., Kaelin A.,"Subband signal processing for hearingaids", In Proc. IEEE Int. Symp. Circuits Syst., 3, pp. 29-32, 1999.

[8] S.Wyrsch and A. Kaelin, "Subband signal processing for hearing aids,"in Proc. IEEE Int. Symp. Circuits Syst., Orlando, FL, Jul. 1999, vol. 3,pp. 29-32.

[9] K. M. Kates and K. H. Arehart, "Multichannel dynamic-range compression using digital frequency warping," EURASIP J. Appl. Signal Process., vol. 18, no. 1, pp. 3003-3014, Jan. 2005.

[10] R. Brennan and T. Schneider, "A flexible filter bank structure for extensive signal manipulations in digital hearing aids," in Proc. IEEE Int. Symp. Circuits Syst., Monterey, CA, Jun. 1998, vol. 6, pp. 569-572.

[11] M. Harteneck, S. Weiss, and R. W. Stewart, "Design of near perfect reconstruction oversampled filter banks for subband adaptive filters", IEEE Trans. Circuits Syst., vol. 46, no. 8, pp. 1081-1086, Aug. 1999.
[12] M. B. Sachs, I. C. Bruce, R. L. Miller, and E. D. Young, "Biological basis of hearing-aid design," Ann. Biomed. Eng., vol. 30, no. 2, pp. 157168, Feb. 2002.

[13] D. Bustamante, T.Worrall, and M. Williamson, "Measurement of adaptive suppression of acoustic feedback in hearing aids," in Proc. 1989IEEE ICASSP, 1989, pp. 2017-2020.

[14] J. Kates, "Feedback cancellation in hearing aids: Results from a computer simulation," IEEE Trans. Signal Processing, vol. 39, pp. 553562,1991 .

[15] J. Maxwell and P. Zurek, "Reducing acoustic feedback in hearing aids,"IEEE Trans. Speech Audio Processing, vol. 4, pp. 304-313, July 1995.

[16] P. Estermann and A. Kaelin, "Feedback cancellation in hearing aids Results from using frequency-domain adaptive filters," in Proc. 1994 IEEE ISCAS, 1994, pp. 257-260.

[17] A. Oppenheim and R. Schaffer, Discrete-Time Signal Processing.Englewood-Cliffs, NJ: Prentice-Hall, 1989.

[18] Agnew, J., 1993. Application of a notch filter to reduce acoustic feedback. Hearing J. 46, 37-43.

[19] Bustamante, D.K., Worral, T.L., Williamson, M.J., 1989.Measurement of adaptive suppression of acoustic feedbackin hearing aids. In: Proc. IEEE ICASSP-89, pp. 2017-2020.

[20] Chi, H.F., 1999. Adaptive feedback cancellation for hearing aids: Theories, algorithms, computations, and systems. Ph.D. dissertation, Department of Electrical Engineering, University of California, Los Angeles.

[21] Chi, H.F., Gao, S.X., Soli, S.D., 1999. A novel approach ofadaptive feedback cancellation for hearing aids. In: Proc.IEEE ISCAS-99, pp. 187-190.

[22] Cox, R.M., 1982. Combined effects of earmold vents and suboscillatory feedback on hearing aid frequency response.Ear Hearing 3, 12-17.

[23] Dillon, H., 1991. Allowing for real ear venting effects when selecting the coupler gain of hearing aids. Ear Hearing 12,406-416.

[24] B. Gold, "Robust speech processing," M.I.T. Lincoln Lab., Tech. Note 1976-6, DDC AD-A012 P99/0, Jan. 27, 1976.

[25] Schneider, T., Brennan R.L., "A Multichannel Compression Strategy for a Digital Hearing Aid," Proc. ICASSP-97, Munich, Germany, pp. 411415.

[26] B. Saha, s. Khan, c. Shahnaz, s. A. Fattah, m. T. Islam and a. I. Khan, "configurable digital hearing aid system with reduction of noise for speech enhancement using spectral subtraction method and frequency dependent amplification," tencon 2018 - 2018 ieee region 10 conference 2018, pp. 0735-0740, doi: 10.1109/tencon.2018.8650450.

[27] N. M. Yunus, n. A. Noor affande, r. M. Ramli, a. O. A. Noor and s. A. Samad, "preprocessing noise reduction for assistive listening system," 2021 1st international conference on electronic and electrical engineering and intelligent system (ice3is), 2021, pp. 114-119, doi: 10.1109/ice3is54102.2021.9649710. 